\title{
A nomogram to predict the progression-free survival of clival chordoma
}

\author{
${ }^{*}$ Yixuan Zhai, MD,,2 Jiwei Bai, MD,,3,4 Mingxuan Li, MD,1 Shuai Wang, MD,, Chuzhong Li, MD,,3-5 \\ Xinting Wei, MD, ${ }^{2}$ and Yazhuo Zhang, MD, $\mathrm{PhD}^{1,3-5}$ \\ 1Beijing Neurosurgical Institute, Capital Medical University, Beijing; ${ }^{2}$ Department of Neurosurgery, The First Affiliated Hospital of \\ Zhengzhou University, Zhengzhou; ${ }^{3}$ Department of Neurosurgery, Beijing Tiantan Hospital, Capital Medical University, Beijing; \\ ${ }^{4}$ China National Clinical Research Center for Neurological Diseases, Beijing; and ${ }^{5}$ Beijing Institute for Brain Disorders Brain \\ Tumor Center, Beijing, China
}

OBJECTIVE Chordoma shows poor patient prognosis because of its high recurrence rate. Even though many clinical factors and biomarkers are reported to be associated with prognosis, no prediction model has been applied clinically. Thus, the authors aim to derive and validate a prognostic nomogram to predict progression-free survival (PFS) of chordoma.

METHODS A total of 201 patients were randomly divided into a derivation group (151 cases) and a validation group (50 cases). The expression levels of biomarkers were quantified using tissue microarray analysis. A nomogram was established via univariate and multivariate Cox regression analysis in the derivation group. The predictive performance of the nomogram was then tested in the validation group.

RESULTS The mean follow-up interval was 57 month (range 26-107 months). One clinical factor and 3 biomarkers were confirmed to be associated with PFS, including degree of resection, E-cadherin, Ki-67, and VEGFA. The nomogram with these prognostic factors had areas under the receiver operating characteristic curve of 0.87 and 0.95 in the derivation group at 3 years and 5 years, respectively, compared with 0.87 and 0.84 in the validation group. Calibration and score-stratified survival curve were good in the derivation group and validation group, respectively.

CONCLUSIONS The established nomogram performs well for predicting the PFS of chordoma and for risk stratification, which could facilitate prognostic evaluation and follow-up.

https://thejns.org/doi/abs/10.3171/2019.10.JNS192414

KEYWORDS chordoma; progression-free survival; nomogram; oncology

$C_{1}$ HORDOMA is a rare bone tumor with the characteristics of aggressive growth and poor prognosis, and is considered to arise from notochordal remnants. ${ }^{17}$ The annual incidence of the tumor is close to one case per million people, which accounts for $1 \%-4 \%$ of all malignant bone tumors. ${ }^{13}$ The tumor mainly locates in the axial skeleton: almost $50 \%$ are found in the sacrococcygeal region, followed by the clival region (35\%) and mobile spine $(15 \%) .{ }^{20}$ It is pathologically classified into three types: conventional, chondroid, and dedifferentiated. ${ }^{6}$

Chordoma is not sensitive to chemotherapy and radiotherapy. ${ }^{15}$ Targeted drugs do not show satisfactory effect either. Thus, gross-total resection (GTR) is still the first choice of neurosurgeons. However, the tendency of chordomas to adhere to and infiltrate into important structures make it difficult to achieve GTR, resulting in high recurrence rates. ${ }^{16}$ So far, there has been no study reporting a method for predicting the prognosis of clival chordoma. A nomogram is a clinically practical prediction model that has been used in many tumors, such as prostate and gastric cancers. ${ }^{9}$ Use of a nomogram could help doctors make clinical decisions and effectively follow-up patients, which would improve the prognosis. ${ }^{10}$ Therefore, establishing a nomogram to predict the prognosis of clival chordoma could be very useful, and predicting the prognosis is critical to both neurosurgeons and patients for treatment deci-

ABBREVIATIONS AUC = area under the ROC curve; $\mathrm{GTR}=$ gross-total resection; $\mathrm{HR}=$ hazard ratio; $\mathrm{OS}=$ overall survival; $\mathrm{PFS}=$ progression-free survival; $\mathrm{PI}=$ prognostic index; PR = partial resection; ROC = receiver operating characteristic; $S T R=$ subtotal resection; TMA = tissue microarray

SUBMITTED September 3, 2019. ACCEPTED October 25, 2019.

INCLUDE WHEN CITING Published online December 27, 2019; DOI: 10.3171/2019.10.JNS192414.

${ }^{*} \mathrm{X}$. Wei and $\mathrm{Y}$. Zhang contributed equally to this work. 
sion-making. The aim of our study is to create and validate a nomogram to predict progression-free survival (PFS) of clival chordoma.

\section{Methods \\ Study Population}

A total of 201 patients who underwent an operation using an endonasal endoscopic approach or craniotomy approach between January 2008 and December 2014 at Beijing Tiantan Hospital were included in the study. The inclusion criteria were as follows: 1) the lesions were located in the clival region, 2) the histopathological diagnosis was chordoma, 3) medical records were complete, and 4) the follow-up interval was at least 24 months. Exclusion criteria were as follows: 1) tumor specimen was insufficient for building the tissue microarray (TMA), 2) medical records were incomplete, 3 ) and patients were lost to follow-up. The medical ethics committee of Beijing Tiantan Hospital approved the study.

The degree of resection was determined by comparing postoperative with preoperative imaging, and divided into three categories: gross-total resection (GTR; no residual tumor in the postoperative imaging), subtotal resection (STR; $<5 \%$ residual tumor), and partial resection (PR; > $5 \%$ residual tumor). ${ }^{4}$ Tumor volume was estimated by using the Coniglobus formula ${ }^{3}$ (length $\times$ width $\times$ height $/ 2$ ).

\section{Immunohistochemical Staining and Analysis}

Formalin-fixed, paraffin-embedded chordoma specimens were used to build a TMA. Sections ( $4 \mu \mathrm{m}$ thick) were cut from each TMA. The automated immunohistochemical staining system (Leica BOND III) was used to perform immuohistochemical staining. The stained TMA slides were scanned by a Leica Aperio AT2 scanner at a magnification of 400x. The digital images were analyzed by Leica Aperio ImageScope software (version 12.3). The H-score was calculated to quantify staining intensity. The H-score $=1 \times($ percentage of weakly positive $)+2 \times$ (percentage of moderately positive) $+3 \times$ (percentage of strongly positive). ${ }^{11}$ Thus, the $\mathrm{H}$-score ranges from 0 to 300 .

\section{Statistical Analysis and Nomogram Construction}

All patients were randomly divided into two groups (ratio of patients in the derivation group to those in the validation group $=3$ to 1 ). The chi-square test or t-test was used to evaluate differences between the two groups. Cox regression analysis (univariate and multivariate) was used to identify the risk factors. First we performed univariate Cox regression analysis using all the clinical and biomarker variables. Then, multivariate Cox regression analysis was performed using variables with $\mathrm{p}$ values < 0.05 in the univariate analysis. The backward mode ${ }^{14}$ was used to select the independent factors, which was used to establish the nomogram.

A nomogram was formulated based on the results of multivariate Cox regression analysis in the derivation group. To evaluate the nomogram performance, the area under the receiver operating characteristic (ROC) curve (AUC) was used to evaluate the sensitivity and specificity of the nomogram, ${ }^{7}$ and a calibration curve was drawn as an indicator of internal calibration..$^{5}$ Regarding the AUC, a value $>0.7$ indicates a good discrimination. The nomogram performance was then further evaluated in the validation group by ROC and calibration curve. Lastly, according to the nomogram, we calculated the risk score for every patient. Patients were divided into two groups (high-risk vs low-risk) by the mean score. The PFS was compared between the high-risk and low-risk groups using the Kaplan-Meier method. The biomarker expression levels were also compared between the two groups.

Statistical analyses were performed using the R statistical program (version 3.5.1). The following packages were used during analysis: "survival" (Terry M. Therneau), "plyr" (Hadley Wickham), "rms" (Frank E. Harrell), and "survminer" (Alboukadel Kassambara). A p value $<0.05$ was defined as statistically significant.

\section{Results \\ Patient Summary}

A total of 201 patients (111 men and 90 women) were included in the study. These patients harbored 149 primary and 52 recurrent chordomas. The mean patient age was 41.02 years (range 3-78 years). The mean duration of medical history was 19.24 months (range $0.5-120$ months). Tumor volume ranged from $1.44 \mathrm{~cm}^{3}$ to $210 \mathrm{~cm}^{3}$ (mean $33.36 \mathrm{~cm}^{3}$ ). According to the Al-Mefty classification, ${ }^{1}$ there were 28,97 , and 76 cases of type I, type II, and type III chordomas, respectively. TR was achieved in 64 cases (31.8\%), STR in 66 cases (32.8\%), and PR in 71 cases $(35.4 \%)$. There are 89 patients $(44.3 \%)$ receiving postoperative radiotherapy. The mean follow-up interval was 57 months (range 26-107 months).

The patients were randomly divided into two groups (derivation group, $\mathrm{n}=151$ cases; validation group, $\mathrm{n}=50$ cases). Patient characteristics are shown in Table 1, which were similar overall between the derivation and validation groups.

\section{Univariate and Multivariate Analysis}

Based on literature reviews and previous results from our research team, we focused on 8 clinical factors and 50 biomarkers that could be prognostic factors. Then, Cox regression analysis was performed in the derivation group to determine the potential prognostic factors. The median PFS was 24 months. The PFS rates at 3 and 5 years were $41.8 \%$ and $27.9 \%$, respectively.

Univariate Cox regression analysis was used to explore the association between prognosis and each clinical factor and biomarker. Among 8 clinical factors, 3 factors (GTR, Al-Mefty type I, and primary chordoma) were significantly favorable clinical factors. Among 50 biomarkers, 8 (Ki-67, E-cadherin, Ras, NF-KB, BRCA2, Caspase-8, VEGFA, and SFRP4) were significant prognostic biomarkers (Fig. 1).

Multivariate Cox regression analysis was performed among clinical factors and candidate biomarkers, respectively. We found that among the 3 significant clinical factors in univariate Cox regression, degree of resection was the only statistically significant independent prognostic factor. Among the 8 candidate biomarkers, 3 biomarkers 
TABLE 1. Patient characteristics

\begin{tabular}{|c|c|c|c|}
\hline Characteristic & Derivation Group & Validation Group & $p$ Value \\
\hline No. of patients (\%) & $151(75.1)$ & $50(24.9)$ & \\
\hline Sex, n (\%) & & & 0.97 \\
\hline Male & $84(55.6)$ & $27(54.0)$ & \\
\hline Female & $67(44.4)$ & $23(46.0)$ & \\
\hline Mean age (range), yrs & $41.89(8-78)$ & $38.38(3-69)$ & 0.17 \\
\hline Mean duration of history (range), mos & $20.62(1-120)$ & $15.09(0.5-96)$ & 0.23 \\
\hline Mean volume (range), $\mathrm{cm}^{3}$ & $32.54(1.74-180.31)$ & $35.82(1.44-210)$ & 0.59 \\
\hline Al-Mefty classification, $\mathrm{n}(\%)$ & & & 0.12 \\
\hline Type I & $25(16.6)$ & $3(6.0)$ & \\
\hline Type II & $73(48.3)$ & $24(48.0)$ & \\
\hline Type III & $53(35.1)$ & $23(46.0)$ & \\
\hline Primary tumor, $\mathrm{n}(\%)$ & $110(72.8)$ & $39(78.0)$ & 0.59 \\
\hline Recurrent tumor, n (\%) & $41(27.2)$ & $11(22.0)$ & \\
\hline Degree of resection, $\mathrm{n}(\%)$ & & & 0.15 \\
\hline GTR & $49(32.5)$ & $15(30.0)$ & \\
\hline STR & $54(35.8)$ & $12(24.0)$ & \\
\hline PR & $48(31.7)$ & $23(46.0)$ & \\
\hline Postop radiotherapy, n (\%) & & & $>0.99$ \\
\hline Yes & $67(44.4)$ & $22(44.0)$ & \\
\hline No & $84(55.6)$ & $28(56.0)$ & \\
\hline Complications, n (\%) & & & $>0.99$ \\
\hline Yes & $67(44.4)$ & $22(44.0)$ & \\
\hline No & $84(55.6)$ & $28(56.0)$ & \\
\hline
\end{tabular}

(Ki-67, E-cadherin, and VEGFA) were independent prognostic factors. Table 2 presents the results of univariate and multivariate Cox regression analysis among clinical factors and candidate biomarkers in the derivation group.

Based on the clinical factors and biomarkers, we established a PFS prediction model in the derivation group. The prognostic index (PI) was calculated by the following equation: $\mathrm{PI}=0.8904 \times$ degree of resection +0.0291 $\times$ E-cadherin score $+0.0447 \times$ Ki-67 score $+0.0035 \times$ VEGFA score. The higher the PI was, the worse the prognosis would be.

\section{Nomogram Derivation and Validation}

A nomogram (Fig. 2) representing the prognostic model was derived to make it easier to use clinically. The estimated 3- and 5-year PFS rates were obtained according to the sum of each variable score. The ROC curve was used to evaluate the sensitivity and specificity of the nomogram. The model showed a good sensitivity and specificity with AUCs of 0.87 and 0.95 for PFS prediction at 3 and 5 years, respectively (Fig. 3A). The calibration graph was drawn with the predicted 3-year PFS rate and corresponding observed PFS rate. It showed a good calibration of the nomogram (Fig. 3C).

According to the established nomogram, the score of each patient in the derivation group was calculated. Furthermore, the derivation group was divided into two groups by the mean score (cutoff value 105): a low-risk group (lower than the mean score of the group) and a highrisk group (higher than the mean score of the group). The median PFS of the low-risk group was 48 months, which was significantly higher than that of the high-risk group (12 months, $p<0.0001$; Fig. 4A). The mean Ki-67 positive rate of the low-risk group in the derivation dataset was $9.79 \%$, which was significantly lower than that of high-risk group $(19.60 \%, \mathrm{p}<0.0001$; Table 3$)$.

To validate the nomogram's stability, we conducted a validation study using a validation group. The AUCs of the nomogram in the validation group were 0.87 and 0.84 for PFS prediction at 3 years and 5 years, respectively, demonstrating a good prediction (Fig. 3B). The calibration graph of the validation group showed a moderate calibration (Fig. 3D). The validation group was also divided into two groups (low-risk and high-risk groups) by the same cutoff value of the derivation group (cutoff value $=105$ ). The median PFS values of the low-risk and high-risk groups were 42 and 12 months $(\mathrm{p}=0.0002)$, respectively. The score-stratified survival curve showed satisfactory stratification (Fig. 4B). The mean Ki-67 positive rate of the low-risk group in the validation dataset was $8.74 \%$, which was significantly lower than that of the high-risk group $(18.42 \%, \mathrm{p}=0.0002$; Table 3).

\section{Discussion}

Chordoma has a poor prognosis because of a high recurrence rate. It has been reported that the 5-year rates 
Zhai et al.
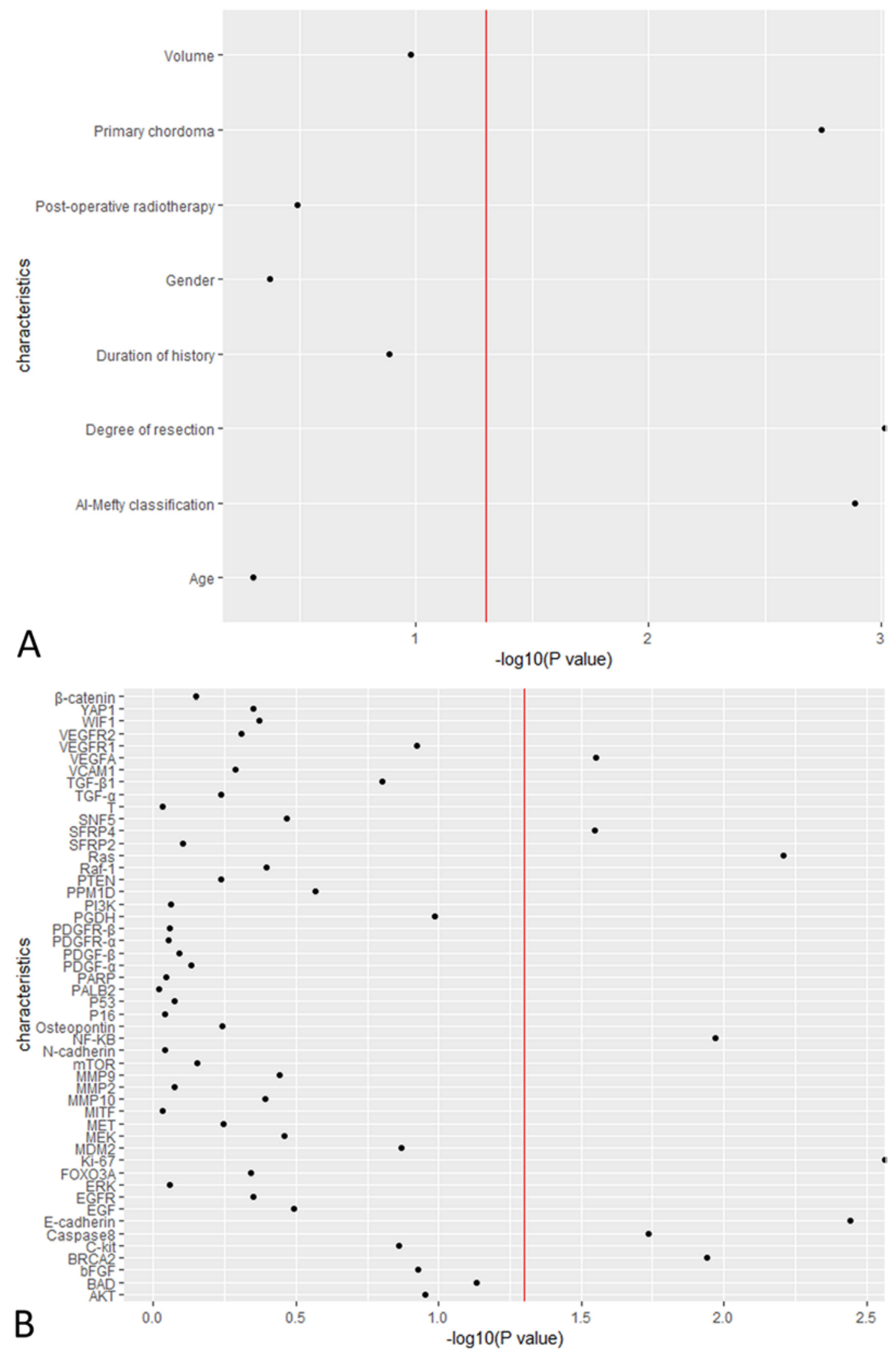

FIG. 1. Univariate Cox regression analysis of 8 clinical factors (A) and 50 biomarkers (B). Scatter diagram showed that 3 clinical factors and 8 biomarkers were statistically significant. The red vertical line represents a $p$ value of 0.05 . Figure is available in color online only. 
TABLE 2. Univariate and multivariate Cox regression analysis

\begin{tabular}{|c|c|c|c|c|}
\hline \multirow[b]{2}{*}{ Variable } & \multirow{2}{*}{$\begin{array}{l}\text { Univariate Analysis } \\
\text { (p Value) }\end{array}$} & \multicolumn{3}{|c|}{ Multivariate Analysis } \\
\hline & & $\mathrm{HR}$ & $95 \% \mathrm{Cl}$ & $p$ Value \\
\hline \multicolumn{5}{|l|}{ Clinical factors } \\
\hline Age & 0.498 & & & \\
\hline Sex (female vs male) & 0.4223 & & & \\
\hline Duration of history & 0.1294 & & & \\
\hline Volume & 0.1055 & & & \\
\hline Degree of resection (PR or STR vs GTR) & $<0.0001$ & 2.7167 & $1.9413-3.802$ & $<0.0001$ \\
\hline Al-Mefty classification (type II or type III vs type I) & 0.0013 & NS & & \\
\hline Recurrent vs primary chordoma & 0.0018 & NS & & \\
\hline With vs without postop radiotherapy & 0.3234 & & & \\
\hline \multicolumn{5}{|l|}{ Biomarkers } \\
\hline Ki-67 expression level & $<0.0001$ & 1.0285 & $1.0107-1.047$ & 0.0016 \\
\hline E-cadherin expression level & 0.0036 & 1.0231 & $1.0081-1.038$ & 0.0024 \\
\hline Ras expression level & 0.0062 & NS & & \\
\hline NF-KB expression level & 0.0107 & NS & & \\
\hline BRCA2 expression level & 0.0115 & NS & & \\
\hline Caspase-8 expression level & 0.0183 & NS & & \\
\hline VEGFA expression level & 0.0281 & 1.0079 & $1.0027-1.013$ & 0.0029 \\
\hline SFRP4 expression level & 0.0284 & NS & & \\
\hline
\end{tabular}

$\mathrm{Cl}=$ confidence interval; $\mathrm{NS}=$ predictors nonselected with the backward method.

for PFS and overall survival (OS) were 59.2\% and 77.3\%, respectively. ${ }^{22}$ Postoperative enhanced MRI is the most widely used evaluation technique in follow-up. There are, as far as we know, many prognostic factors that have been reported, such as degree of resection, radiotherapy, ${ }^{2}$ SNF5, PDGFR- $\beta,{ }^{21}$ PARP1, etc. However, few prediction models have been established in the published studies, which is inconvenient for early diagnosis or regrowth monitoring. Therefore, we decided to establish an efficient prediction model to predict regrowth of clival chordoma using clinical factors and biomarkers.

Many articles have reported clinical factors associated with PFS, but no consensus has been reached in this respect. As has been consistently shown in previous studies, ${ }^{12,15}$ radical resection can significantly improve a patient's prognosis. Among the clinical factors in this study, degree of resection was the only independent prognostic factor, which is the most important prognostic factor (haz-

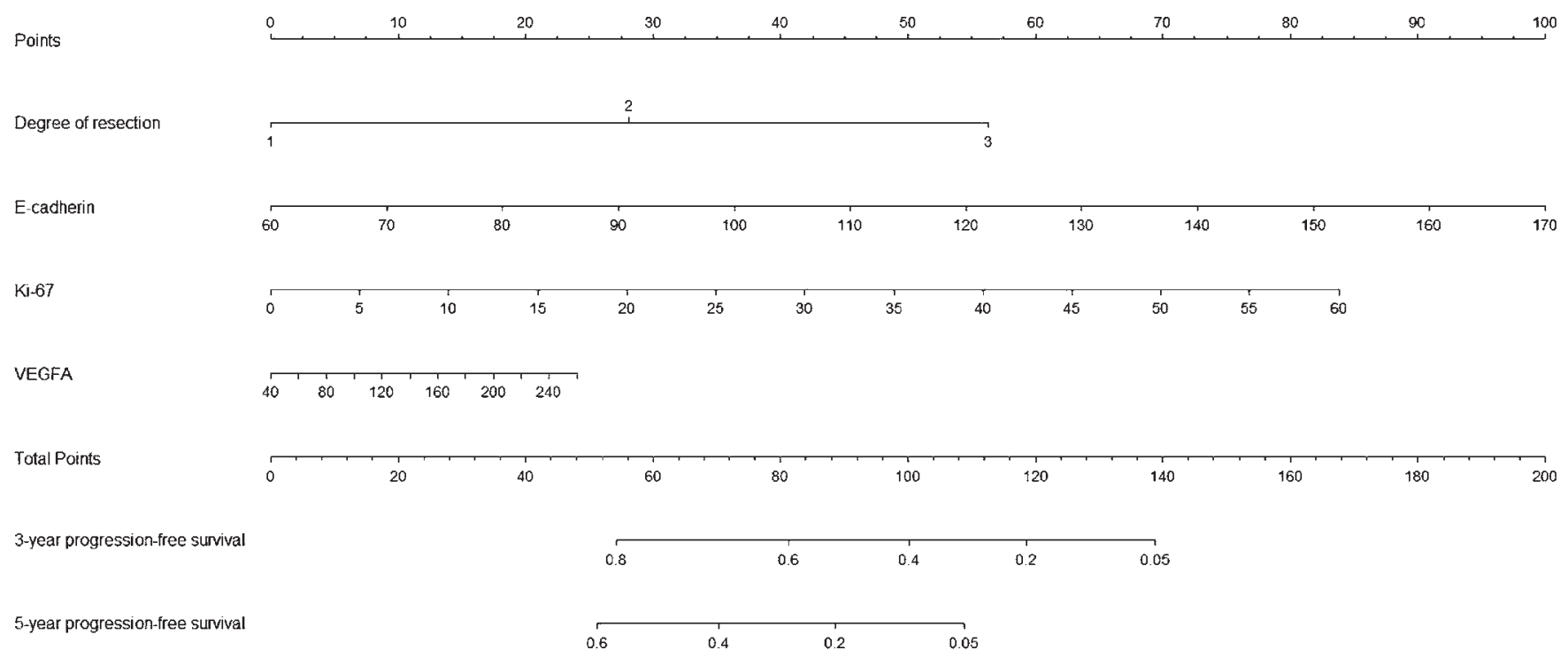

FIG. 2. Nomogram for 3- and 5-year PFS in patients with clival chordomas. 

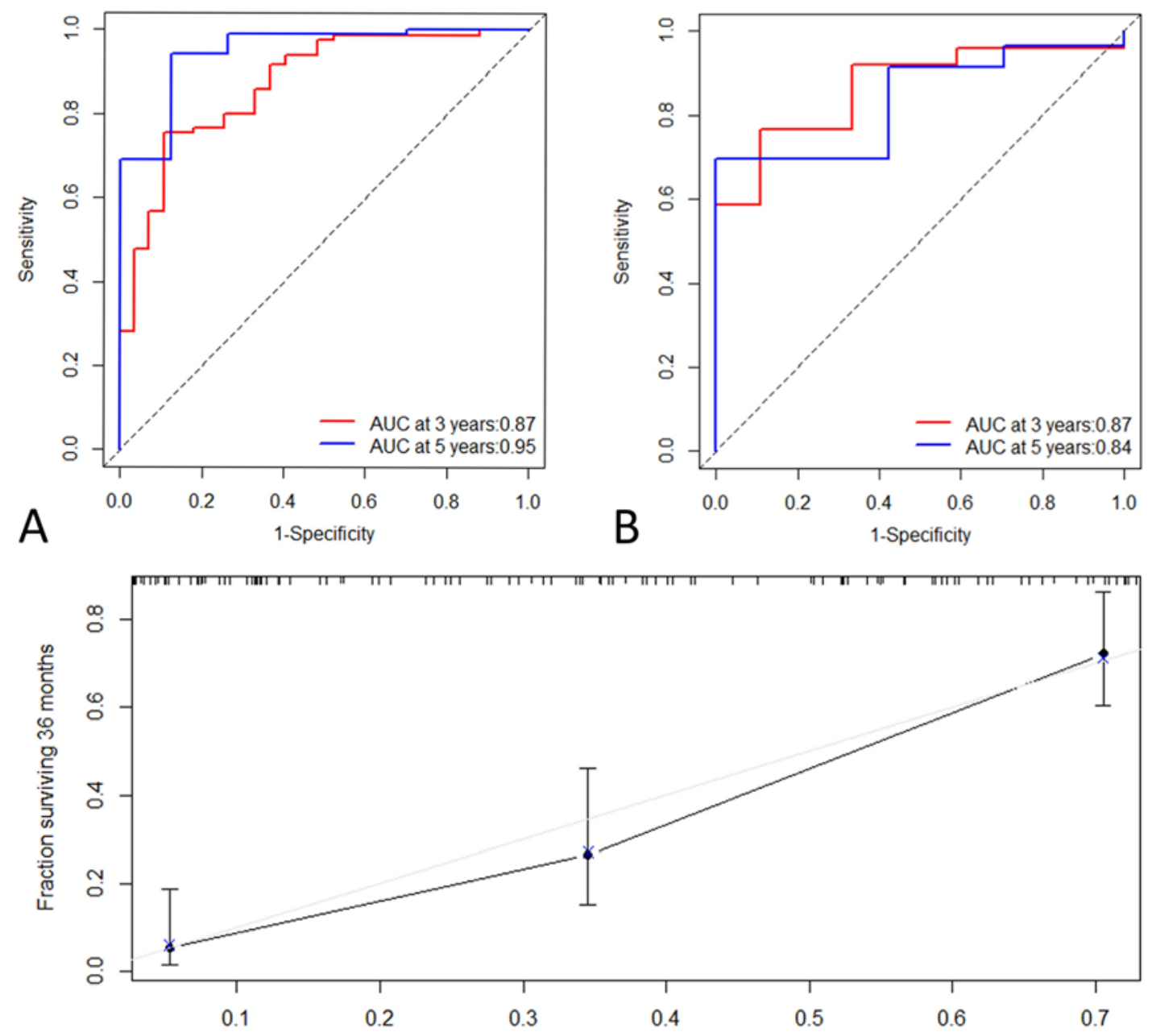

C $\quad n=151 d=100 p=4,50$ subjects per group

Predicted 36 months survival

$X$ - resampling optimism added, $B=1000$ Based on observed-predicted

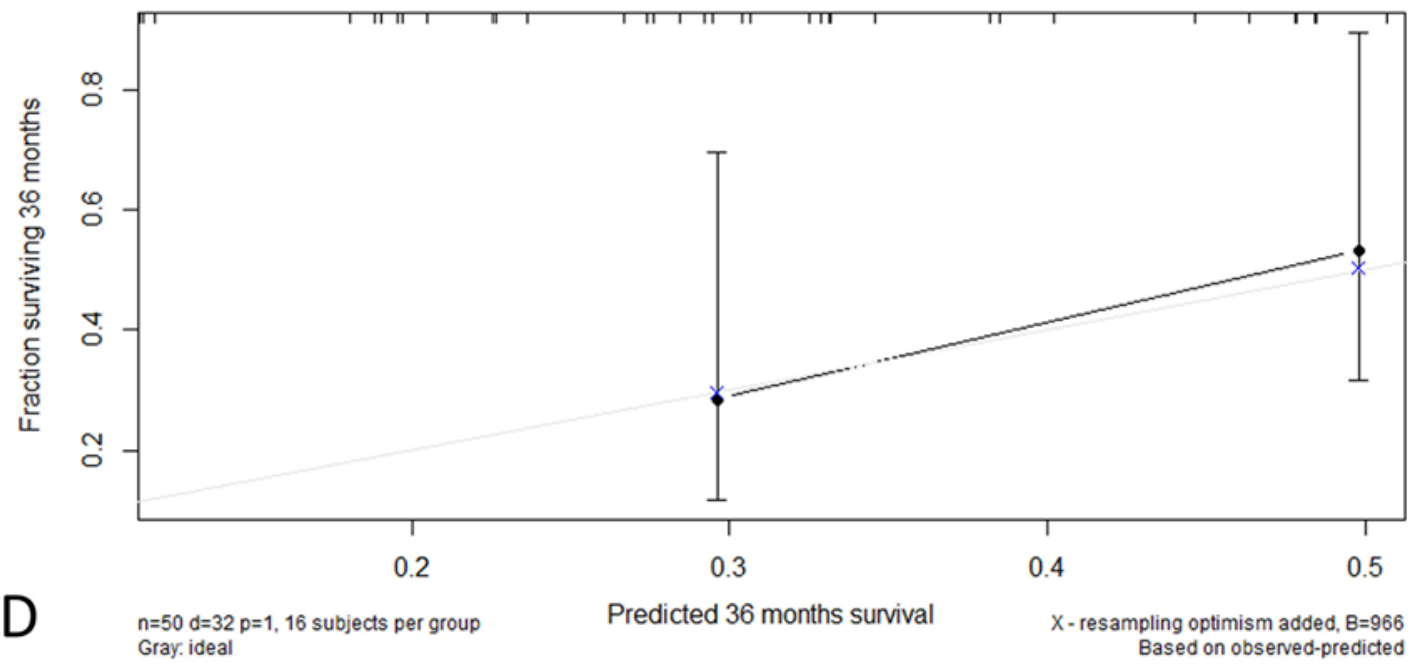

FIG. 3. A and B: ROC curves of the nomogram in the derivation $(A)$ and validation $(B)$ groups. $C$ and $D$ : Calibration of the nomogram in the derivation $(C)$ and validation $(D)$ groups. Figure is available in color online only. 

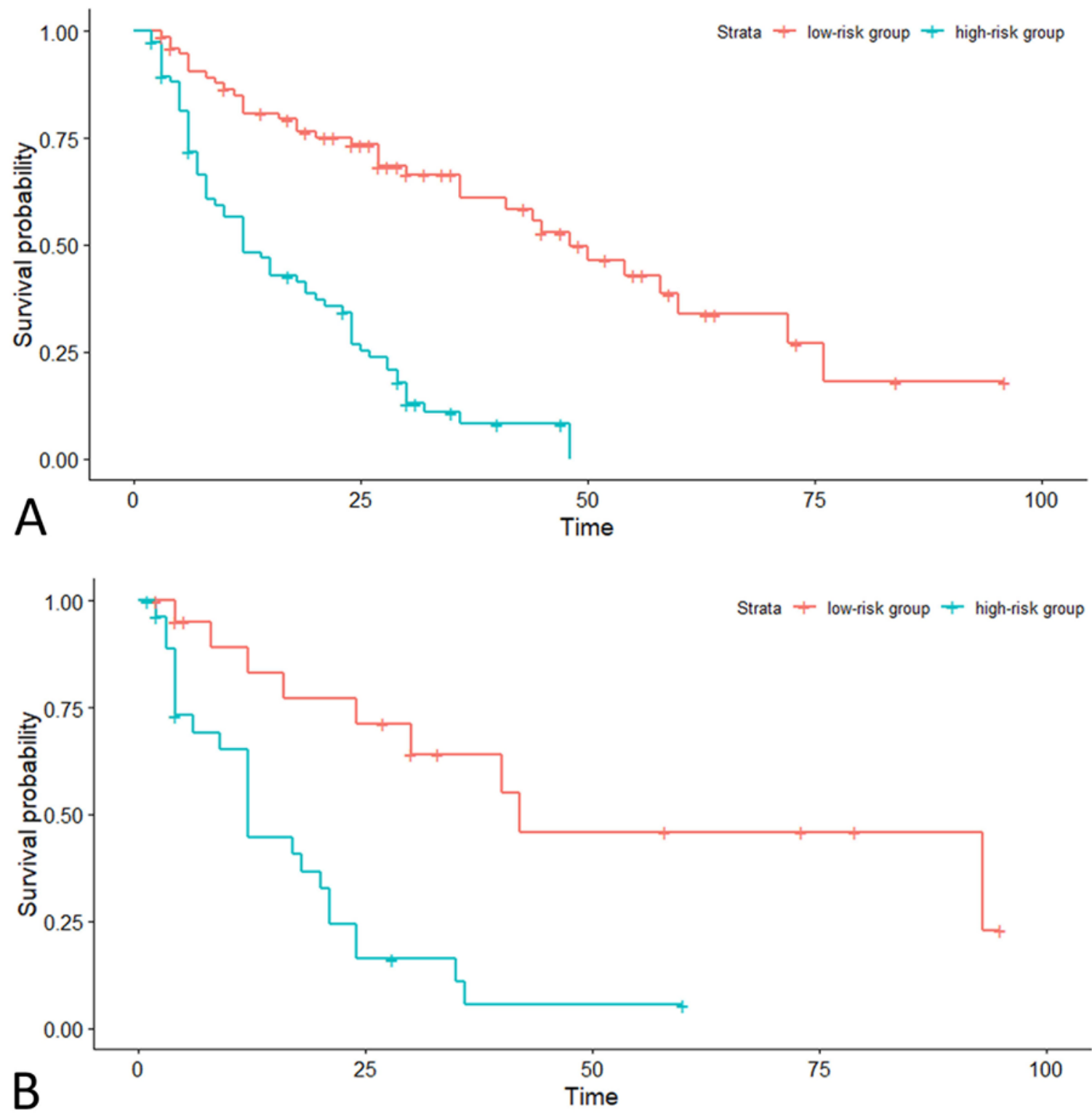

FIG. 4. Kaplan-Meier survival curve of patients in the derivation $(\mathbf{A}, p<0.0001)$ and validation $(\mathbf{B}, p<0.0001)$ groups stratified by nomogram-predicted score. In the low-risk group, the score is lower than the mean score of the group; in the high-risk group, the score is higher than the mean score of the group. Figure is available in color online only.

ard ratio $[\mathrm{HR}]=2.7167)$. With the assistance of intraoperative navigation and Doppler ultrasonography, the neurosurgeon can recognize important structures surrounding the tumors, which improves the GTR rate without increasing the complication rate. Thus, GTR with protection of surrounding structures is the first choice of neurosurgeons.

Radiotherapy, especially proton therapy, has been reported to improve local control and PFS of chordoma. ${ }^{2}$ In our study, the multivariate Cox regression analysis showed that radiotherapy has no relation with PFS. This indicates that chordoma is resistant to radiotherapy, and more studies are needed to explore its role in prolonging PFS.

The Al-Mefty classification is an important factor during the preoperative assessment, ${ }^{1}$ which reveals the dif- ficulty of resection. However, it was not an independent prognostic factor in our study. We speculate that this classification affects patients' PFS through affecting the resection rate.

Ki-67 locates in the nucleus, which is positive in proliferating cells, and shows the proliferative activity of cells. ${ }^{18}$ In our previous study, the average OS in the low Ki-67 expression group was significantly longer than that in the high Ki-67 expression group. ${ }^{19}$ Karamchandani et al. ${ }^{8}$ also found that a chordoma with high $\mathrm{Ki}-67$ expression would recur within 2 months. VEGFA and E-cadherin expressions are prognostic markers in oral squamous cell carcinoma, hepatocellular carcinoma, clear cell renal cell carcinoma, and others. However, few articles have reported the 
TABLE 3. Comparisons between the low-risk and high-risk groups

\begin{tabular}{|c|c|c|c|c|c|c|}
\hline \multirow[b]{2}{*}{ Variable } & \multicolumn{3}{|c|}{ Derivation Group } & \multicolumn{3}{|c|}{ Validation Group } \\
\hline & Low-Risk* & High-Risk* & p Value & Low-Risk* & High-Risk* & $p$ Value \\
\hline Mean $\mathrm{H}$-score of E-cadherin $\pm \mathrm{SD}$ & $104.03 \pm 9.26$ & $114.36 \pm 15.72$ & $<0.0001$ & $105.29 \pm 9.14$ & $112.51 \pm 15.27$ & 0.044 \\
\hline Mean Ki-67 index \pm SD & $9.79 \% \pm 8.51 \%$ & $19.60 \% \pm 12.29 \%$ & $<0.0001$ & $8.74 \% \pm 6.08 \%$ & $18.42 \% \pm 10.53 \%$ & 0.0002 \\
\hline Mean $\mathrm{H}$-score of VEGFA \pm SD & $140.64 \pm 39.28$ & $155.30 \pm 46.01$ & 0.037 & $134.05 \pm 38.43$ & $166.10 \pm 50.36$ & 0.014 \\
\hline Mean score \pm SD $†$ & $77.07 \pm 17.97$ & $132.72 \pm 18.43$ & $<0.0001$ & $77.73 \pm 16.72$ & $135.49 \pm 22.86$ & $<0.0001$ \\
\hline Median PFS, mos & 48 & 12 & $<0.0001$ & 42 & 12 & 0.0002 \\
\hline
\end{tabular}

* Divided by the same cutoff value (105), which was the mean score of the derivation group.

† Calculated according to the established nomogram.

prediction value of E-cadherin and VEGFA in chordoma. In our study, we are the first to report that they are independent prognostic factors, which provides a new research direction.

Even though many biomarkers are reported to be associated with prognosis, none of them have been adapted for clinical use because of their low sensitivity and inconvenience. To assist the neurosurgeon in clinical decisionmaking, we developed a nomogram using the clinical factor and biomarkers. The cutoff value derived from the nomogram could obviously divide patients into long and short PFS groups. We consider it a very useful tool to predict the PFS of chordoma, help neurosurgeons make decisions, and stratify patients for follow-up. All of the factors included in the nomogram are objective factors, guaranteeing its stability and wider applicability.

However, our study has some limitations. First, the nomogram needs to be further evaluated by prospective research with larger sample sizes in multiple centers. Second, the role of radiotherapy remains controversial. Some published articles found that radiotherapy, especially proton therapy, could prolong PFS. In contrast, some reported that radiotherapy did not offer a survival benefit. In our study, the Cox regression analysis showed no relationship between radiotherapy and PFS. This may be due to the low proportion of postoperative radiotherapy. Thus, the role of radiotherapy needs to be further studied.

\section{Conclusions}

We found that degree of resection as well as E-cadherin, Ki-67, and VEGFA expression levels were independent prognostic factors of PFS in patients with clival chordoma. The established nomogram, consisting of a clinical factor and biomarkers, provides a novel tool to predict prognosis and performs well for risk stratification. The sensitivity and specificity is good in both the derivation and validation groups.

\section{Acknowledgments}

This work was supported by the National Natural Science Foundation of China (grant no. 81771489) and Beijing Municipal Science \& Technology Commission (grant no. Z171100000117002).

\section{References}

1. Al-Mefty O, Borba LA: Skull base chordomas: a management challenge. J Neurosurg 86:182-189, 1997
2. Baumann BC, Lustig RA, Mazzoni S, Grady SM, O'Malley BW, Lee JYK, et al: A prospective clinical trial of proton therapy for chordoma and chondrosarcoma: feasibility assessment. J Surg Oncol 120:200-205, 2019

3. Diao K, Bian SX, Routman DM, Yu C, Kim PE, Wagle NA, et al: Combination ipilimumab and radiosurgery for brain metastases: tumor, edema, and adverse radiation effects. $\mathbf{J}$ Neurosurg 129:1397-1406, 2018

4. Gui S, Zong X, Wang X, Li C, Zhao P, Cao L, et al: Classification and surgical approaches for transnasal endoscopic skull base chordoma resection: a 6-year experience with 161 cases. Neurosurg Rev 39:321-333, 2016

5. Harrell FE Jr, Lee KL, Mark DB: Multivariable prognostic models: issues in developing models, evaluating assumptions and adequacy, and measuring and reducing errors. Stat Med 15:361-387, 1996

6. Heffelfinger MJ, Dahlin DC, MacCarty CS, Beabout JW: Chordomas and cartilaginous tumors at the skull base. Cancer 32:410-420, 1973

7. Kamarudin AN, Cox T, Kolamunnage-Dona R: Time-dependent ROC curve analysis in medical research: current methods and applications. BMC Med Res Methodol 17:53, 2017

8. Karamchandani J, Wu MY, Das S, Vogel H, Muller P, Cusimano M, et al: Highly proliferative sellar chordoma with unusually rapid recurrence. Neuropathology 33:424-430, 2013

9. Kattan MW, Karpeh MS, Mazumdar M, Brennan MF: Postoperative nomogram for disease-specific survival after an R0 resection for gastric carcinoma. J Clin Oncol 21:3647-3650, 2003

10. Kattan MW, Zelefsky MJ, Kupelian PA, Cho D, Scardino PT, Fuks Z, et al: Pretreatment nomogram that predicts 5-year probability of metastasis following three-dimensional conformal radiation therapy for localized prostate cancer. J Clin Oncol 21:4568-4571, 2003

11. Lavorato-Rocha AM, Anjos LG, Cunha IW, Vassallo J, Soares FA, Rocha RM: Immunohistochemical assessment of PTEN in vulvar cancer: best practices for tissue staining, evaluation, and clinical association. Methods 77-78:20-24, 2015

12. Lv GH, Zou MX, Liu FS, Zhang Y, Huang W, Ye A, et al: Clinicopathological and prognostic characteristics in extraaxial chordomas: an integrative analysis of 86 cases and comparison with axial chordomas. Neurosurgery 85:E527E542, 2019

13. McMaster ML, Goldstein AM, Bromley CM, Ishibe N, Parry DM: Chordoma: incidence and survival patterns in the United States, 1973-1995. Cancer Causes Control 12:1-11, 2001

14. Montero PH, Yu C, Palmer FL, Patel PD, Ganly I, Shah JP, et al: Nomograms for preoperative prediction of prognosis in patients with oral cavity squamous cell carcinoma. Cancer 120:214-221, 2014

15. Samii A, Gerganov VM, Herold C, Hayashi N, Naka T, 
Mirzayan MJ, et al: Chordomas of the skull base: surgical management and outcome. J Neurosurg 107:319-324, 2007

16. Sen C, Triana AI, Berglind N, Godbold J, Shrivastava RK: Clival chordomas: clinical management, results, and complications in 71 patients. J Neurosurg 113:1059-1071, 2010

17. Walcott BP, Nahed BV, Mohyeldin A, Coumans JV, Kahle KT, Ferreira MJ: Chordoma: current concepts, management, and future directions. Lancet Oncol 13:e69-e76, 2012

18. Wen S, Zhou W, Li CM, Hu J, Hu XM, Chen P, et al: Ki-67 as a prognostic marker in early-stage non-small cell lung cancer in Asian patients: a meta-analysis of published studies involving 32 studies. BMC Cancer 15:520, 2015

19. Zhai Y, Bai J, Gao H, Wang S, Li M, Gui S, et al: Clinical features and prognostic factors of children and adolescents with clival chordomas. World Neurosurg 98:323-328, 2017

20. Zhai Y, Bai J, Wang S, Du J, Wang J, Li C, et al: Differences in dural penetration of clival chordomas are associated with different prognosis and expression of platelet-derived growth factor receptor- $\beta$. World Neurosurg 98:288-295, 2017

21. Zhai Y, Bai J, Wang S, Gao H, Li M, Li C, et al: Analysis of clinical factors and PDGFR- $\beta$ in predicting prognosis of patients with clival chordoma. J Neurosurg 129:1429-1437, 2018

22. Zou Y, Neale N, Sun J, Yang M, Bai HX, Tang L, et al: Prognostic factors in clival chordomas: an integrated analysis of 347 patients. World Neurosurg 118:e375-e387, 2018

\section{Disclosures}

The authors report no conflict of interest concerning the materials or methods used in this study or the findings specified in this paper.

\section{Author Contributions}

Conception and design: Zhang, Bai, Wang, Wei. Acquisition of data: Zhai, Bai, M Li. Analysis and interpretation of data: Zhai, M Li. Drafting the article: Zhai. Critically revising the article: Zhai, Wei. Reviewed submitted version of manuscript: Zhai, Bai, M Li, Wang, C Li, Wei. Statistical analysis: Zhai. Administrative/ technical/material support: Zhang, C Li, Wei. Study supervision: Zhang, Wang, C Li, Wei.

\section{Correspondence}

Yazhuo Zhang: Capital Medical University, Beijing, China. zyz2004520@yeah.net. 